\title{
Detection of Cardiac Biomarker Troponin (cTnI) with Enhanced Immune Sensitivity using a Single Monoclonal Antibody
}

\author{
Anul Bozdogan ${ }^{1,2}$, Reham F. El-Kased ${ }^{3 *}$, Vanessa Jungbluth ${ }^{2}$, Wolfgang Knoll ${ }^{1,2}$, Jakub \\ Dostalek $^{2 *}$, Amal Kasry ${ }^{1,4 *}$
}

1 CEST Competence Centre for Electrochemical Surface Technology, 2700 Wiener Neustadt, Austria.

2 Biosensor Technologies, AIT-Austrian Institute of Technology GmbH, Konrad-Lorenz-Straße 24, 3430 Tulln, Austria.

3 Department of Microbiology and Immunology, Faculty of Pharmacy, The British University in Egypt (BUE), El-Sherouk City, Suez Desert Road, Cairo 11837, Egypt.

4 Nanotechnology Research Centre (NTRC), The British University in Egypt (BUE), El-Sherouk City, Suez Desert Road, Cairo 11837, Egypt,

\begin{abstract}
Using an immunoassay in combination with surface plasmon fluorescence spectroscopy, we report for the first time the rapid detection of Troponin I, a cardiac muscle protein that serves as a valuable biomarker for diagnosis of myocardial infarction. We discuss the implementation of i) direct, ii) sandwich, and iii) competitive assay formats, based on surface plasmon resonance and surface plasmon field-enhanced fluorescence spectroscopy (SPFS). In order to elucidate the results, we relate the experiments to orientation-dependent interaction of Troponin I epitopes with respective immunoglobulin G monoclonal and polyclonal antibodies. A limit of detection (LoD) of $19 \mathrm{pM}$, with 45 min readout time, was achieved using single monoclonal antibody that is specific for one epitope. The borderline between normal people and patients is $20 \mathrm{pM}$ to $83 \mathrm{pM} \mathrm{cTnI}$ concentration, and upon the outbreak of acute myocardial infraction (AMI) it can raise to $2 \mathrm{nM}$ and levels at 20 $\mathrm{nM}$ for 6-8 days, therefore the achieved LoD covers most of the clinically relevant range. In addition, the sensor allows for the detection of Troponin I using a single monoclonal antibody, which is highly beneficial in case of detection in real samples, where the protein has a complex form leading to hidden epitopes, thus paving the way towards a system that can improve earlystage screening of heart attacks.
\end{abstract}


Cardiovascular diseases (CVD) refer to several types of conditions that affect the heart and blood vessels, which include acute myocardial infarction (AMI), coronary heart disease (CHD), cerebrovascular disease, peripheral arterial disease, rheumatic heart disease, and congenital heart disease. According to the World Health Organization (WHO), CVD is the leading cause of death worldwide, where it is responsible for $30 \%$ of all deaths globally and WHO estimates that by 2030, 23.6 million people will die from CVD [1]. Low- and middle-income countries often lack the integrated principal healthcare programs for early diagnosis and treatment of people with risk factors compared to people in high-income countries, causing the annual costs of CVD management worldwide to be extremely high. In Egypt, as an example, WHO published in 2014 that CHD deaths reached $23.14 \%$ of total deaths, which ranks Egypt as the 23rd in CHD worldwide [2]. Therefore, there is a strong demand for a robust and economical approach for early diagnosis and prognosis of CVD.

The use of classical CVD biomarkers, such as creatine kinase $(\mathrm{CK})$ and lactate dehydrogenase (LDH), has been restricted due to absence of tissue specificity and sensitivity. In 2000, the European Society of Cardiology and the American College of Cardiology recommended the use of Troponin as a biomarker for the diagnosis of AMI [3], due to the presence of tissue-specific isoforms in cardiac muscle. Troponin complex (cTn) is a heterogenic protein, which plays an important role in the regulation of cardiac muscle contraction. The Troponin complex consists of three subunits: troponin $\mathrm{T}$ (cTnT), troponin I (cTnI) and troponin $\mathrm{C}$ (cTnC). Being expressed only in cardiac tissue, troponins I and $\mathrm{T}$ have been the preferred biomarkers for myocardial infarction for a long time [4]. cTnI is confined inside the heart muscle and it is, therefore, considered to be the standard biomarker for detecting acute myocardial infarction, because it is significantly more specific than other heart markers [5]. Early Troponin I detection would lead to faster diagnosis and consequently the initiation of the correct treatment, which improves the prognosis for patients. It has been demonstrated that testing troponins on patient admission and again after 6 to 12 hours provides better risk stratification and early diagnosis [6]. cTnI levels begin to rise 2-3 h after the myocardial infarction and elevation of its levels can persist for up to 10 days, making it ideal for retrospective diagnosis of infarctions [7-9]. The borderline between normal people and patients is $20 \mathrm{pM}$ to $83 \mathrm{pM}$ cTnI concentration, while after the outbreak of AMI, this concentration can go up to $2 \mathrm{nM}$ within 3-6 h, and levels at about $20 \mathrm{nM}$ for $6-$ 8 days [10].

Several epitopes are identified on the cTnI protein; of which only 6 are used in laboratory research and clinical research (aa. 24-40, aa. 22-43, aa. 41-49, aa. 83-93, aa.87-91, and aa.186-192, respectively). Five of these epitopes are located on the $\mathrm{N}$ terminus, which is considered the leading antigenic region of the recombinant version of cTnI protein (TNNI3 sequence [11-13]). Different diagnostic assays have been designed for the quantitative measurements of cTnI in human blood using monoclonal antibodies, which have found wide-spread clinical applications as diagnostic and therapeutic agents for different diseases [14]. However, this approach lacks robustness and the common reason for the inconsistency between cTnI assay measurements can be attributed to the difference in the epitope specificity of the antibodies used in various assays. In addition, some epitopes are lost as a result of degradation of circulating troponin I, whereas others remain unaltered, resulting in different recoveries by different assays [15]. 
Surface plasmon resonance (SPR) biosensors represent an established technique for rapid detection and interaction analysis of biomolecules on solid surfaces [16-18]. In this technique, resonantly excited surface plasmons are used for probing the capture of specific target analytes from the analyzed sample at the sensor surface. Surface plasmons originate from collective oscillations of the electron density in the metal coupled to an associated electromagnetic field that is confined to the metallic layer or metallic nanostructures deployed at the sensor surface. The analyte binding-induced refractive index change detunes the resonant excitation of surface plasmons and thus is converted to an optical signal. In addition, the resonant excitation of surface plasmons generates an enhanced electromagnetic field intensity, which can be employed for the amplification of weak spectroscopy signals such as scattering [20] and fluorescence [21]. Up to now, the probing with surface plasmons was exploited in a range of biosensors for rapid and sensitive detection of cardiac biomarkers including those based on SPR supported by metallic nanoparticles [22], gold nanoparticle aggregation assay with colorimetric readout [23], surface plasmon-enhanced scattering [24], and localized surface plasmon-enhanced fluorescence [25].

The detection of the full length cTni is usually performed either by polyclonal antibody or multiple monoclonal antibodies specific for several epitopes [26-28], in order to increase the sensitivity and the specificity, which is important in case if the protein is in its complex form.

This work reports, for the first time, on using surface plasmon-enhanced fluorescence (SPFS) assay for the sensitive detection of cTnI at clinically relevant concentrations, using only one monoclonal antibody specific for one epitope. Moreover, it attempts to elucidate the role of the biointerface design by combined SPR and SPFS study in order to optimize the assay performance characteristics. This study is supported by protein modelling in order to visualize the dependence of the epitopes exposure on the protein orientation, which plays a significant role and it may provide important insights into the future development of cTnI immunoassays for accurate screening of CVD at early stage.

\section{RESULTS AND DISCUSSION}

Figure 1 (a-b) shows the recombinant version of the full-length troponin (TNNI3) that was separated using 1D SDS PAGE, confirming a molecular weight of $24 \mathrm{KDa}$. Figure 1(c) shows the Western blot results that confirm the affinity of the polyclonal antibody (antiTNNI3, Goat Polyclonal Antibody) to the fulllength protein.

Figure 1 (d) provides an overview of the biomolecules that are further employed in the cTnI assay with plasmonic biosensor readout including the recombinant version cTnI protein TNNI3 and the respective a-TNNI3 antibodies (in color respective to their binding protein epitopes). The structure of full-length recombinant protein TNNI3 was visualized using bioinformatics methods for predicting the three-dimensional structure of protein molecules based on their amino acid sequences (more details are in the Methods section). Figure 1(e) shows the cTnI TNNI3 protein at different orientations where its epitopes are visualized with different colors, while the size difference between the protein and its antibody is compared in Figure 1 (f). The same bioinformatics method was used to visualize the cTnI protein orientations in case of its affinity binding to mouse monoclonal aTNNI3 antibody (recognizing cTnI epitope aa 87-91) and goat polyclonal antibody a-TNNI3 (recognizing cTnI epitope 30-90), or when coupled to the surface by using the Nterminus, Figure 1 (g, h and i, respectively). In order to bind both monoclonal and polyclonal a-TNNI3 antibodies to the cTnI protein, the 

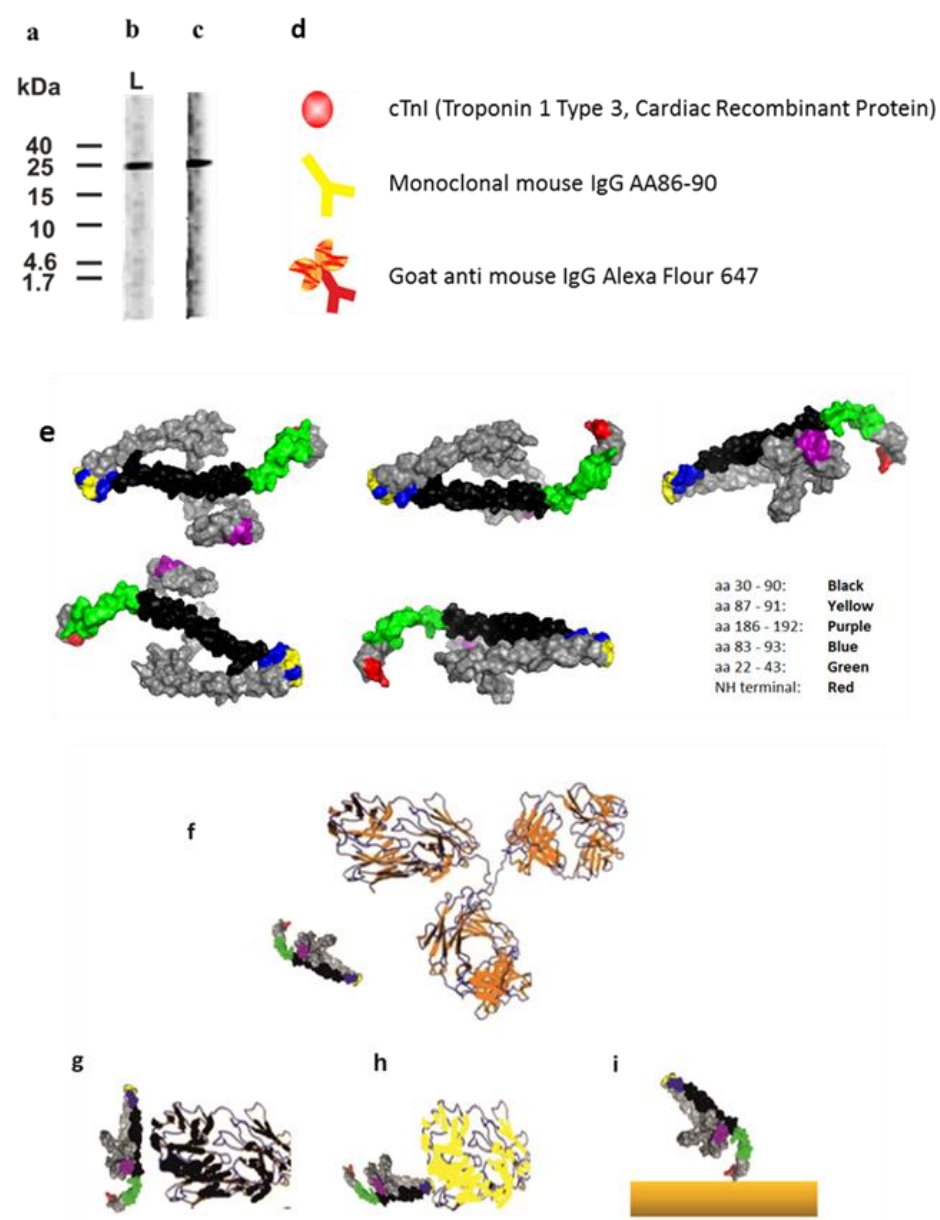

Figure 1. (a) cTnI protein marker lane (kDa), (b) 1D SDS PAGE showing a single band at apparent molecular weight $24 \mathrm{kDa}$ compared to marker lane, (c) Western blot analysis against polyclonal anti-troponin antibody on a polyvinylidene fluoride (PVDF) membrane, (d) a schematic of the cTnI protein and the antibodies used in the experiments with their colors referring to their respective binding epitopes as shown in e, (e) Modeled structure of full-length recombinant TNNI3 version of cTnI protein (aa 1 - 210) using I- TASSER software with its epitopes at different orientations, $(\mathrm{f})$ the size comparison of immunoglobulin $\mathrm{G}$ antibody and $\mathrm{cTnI}(\approx 1: 3)$, (g-i) shows the visualization of the possible cTnI protein orientation according to the binding epitope position; (g) possible orientation of cTnI protein that is affinity bound through its epitope (g) aa $87-91$ and (h) aa $30-90$ to IgG Fab fragment. (i) The immobilization of the cTnI protein to the surface through its N-terminal (red) and the possible protein orientation.

possible protein orientations reveal that the epitope positions (aa 87 - 91 colored in yellow) and (aa 30 - 90 colored in black) spatially overlap, which may prohibit forming a sandwich.
Figure 1 (i) shows that immobilizing the protein directly on the surface, via its $\mathrm{N}$ terminal, allows both these epitope positions (black and yellow) to be exposed, which might allow for efficient binding to respective aTNNI3 antibodies. 

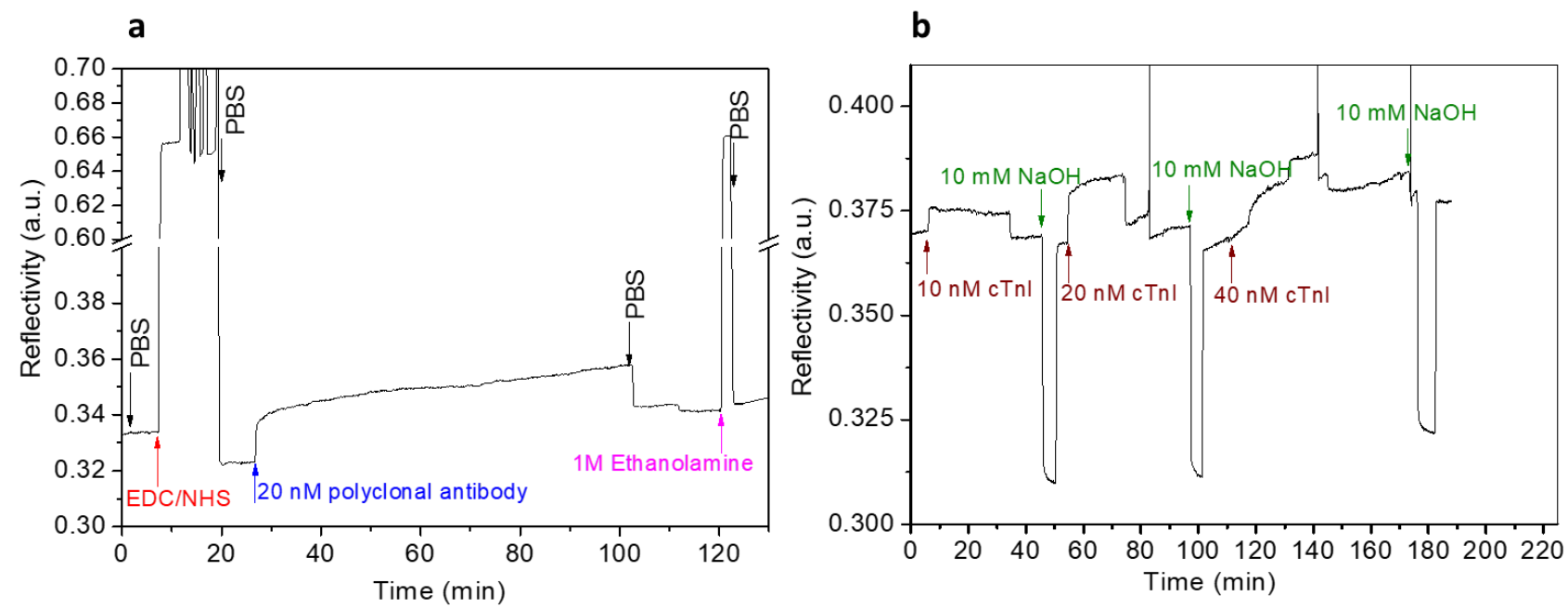

Figure 2. SPR observation of (a) immobilization of polyclonal a-TNNI3 on the surface modified with selfassembled monolayer and (b) the subsequent affinity binding of the cTnI to the tethered polyclonal a-TNNI3 with regeneration steps (10mM NaOH applied) in between flow of cTnI samples.

Based on the Western blot results and the modeling, the cTnI detection was further investigated by using SPR and SPFS. On a first glance, we used the direct label-free SPR method for measuring the affinity binding of the TNNI3 version of cTnI to its polyclonal antibody immobilized on the sensor surface.

To that end, we started with preparing a selfassembled monolayer (SAM), on the sensor gold surface, composed of thiols synthesized with oligo(ethylene glycol) (OEG) and carboxyl groups. Afterwards, the carboxyl groups were activated with a mix of EDC/ NHS [29], followed by the amine coupling of the polyclonal a-TNNI3 antibody via its Nterminus or lysine groups [Figure 2 (a)]. As seen in Figure 2 (b), the binding of cTnI showed measurable SPR signal only at a relatively high concentration of $20 \mathrm{nM}$. cTni is known to have isoelectric point in the range below 7 in case of AMI [30], which means that it would be negatively charged at the neutral $\mathrm{pH}$ that was used in the experiments. Since the gold surface is modified with a mix of thiol SAM carrying with carboxyl group and OEG groups, at $\mathrm{pH} 7$, the surface would be negatively charged leading to repellence of the negatively charged protein, which in turn should prevent nonspecific binding. Although this charge effect is not strong enough to repel all negatively charged proteins, it is enough to avoid nonspecific binding. Since the binding of the cTni wasn't recognized until very high concentration, this wasn't further investigated, and a surface plasmon fluorescence-based protocol was developed.

In order to further confirm that the used monoclonal and polyclonal a-TNNI3 antibodies recognize the cTnI protein and to improve the sensitivity, SPFS readout modality was employed with the use of a secondary antibody conjugated to Alexa Fluor 647 label. The experiment was performed according to the following protocol: 1) cTnI protein was coupled to the sensor surface by the use of amine coupling, 2) a solution with monoclonal mouse or polyclonal goat antibody a-TNNI3 (concentration of 1, 5, and $20 \mathrm{nM}$ ) was rinsed over this surface, followed by 3 ) reaction of the affinity captured a-TNNI3 with secondary anti-mouse or anti-goat antibody, respectively. The secondary antibody was conjugated with Alexa Fluor 647 and dissolved at a concentration of $6 \mathrm{nM}$. As 

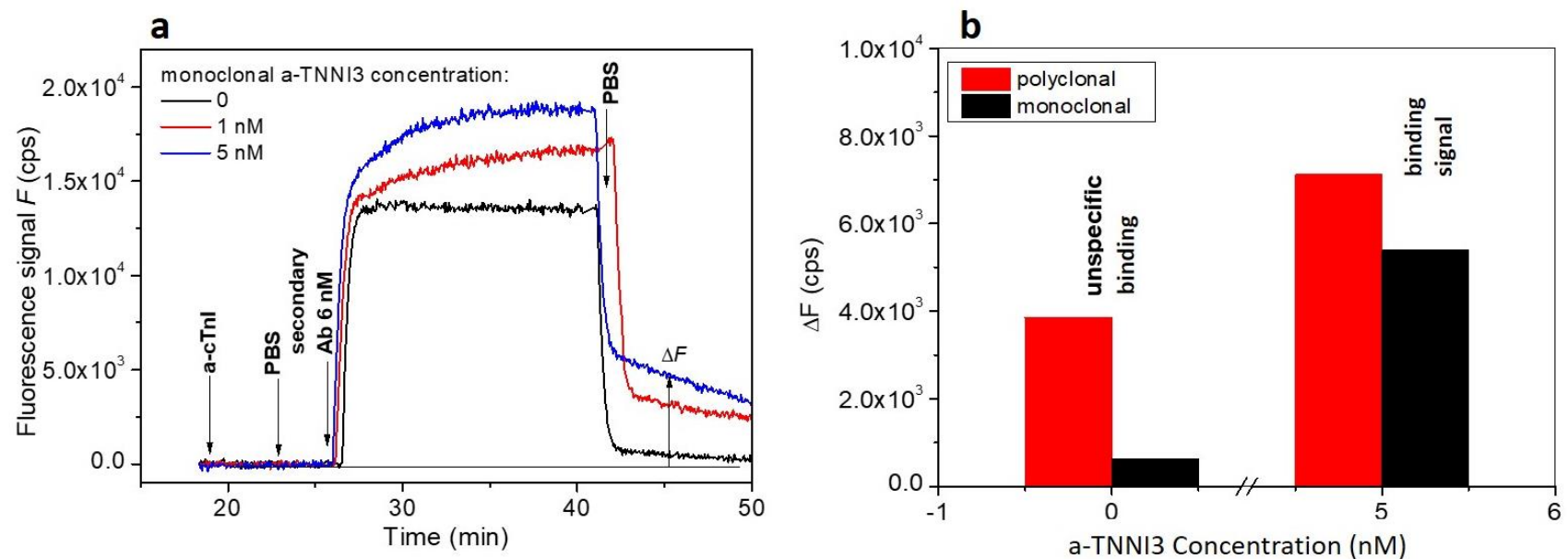

Figure 3. (a) A nonspecific binding test performed by flowing $6 \mathrm{nM}$ labeled secondary antibody specific to the monoclonal one, on the surface modified with the cTnI, no signal was observed after washing the surface (black), followed by binding of the secondary antibody to different concentrations of the monoclonal antibody bound to the cTnI, which gives clear fluorescence signal (red, blue) indicating successful binding. (b) A nonspecific binding test performed by flowing $6 \mathrm{nM}$ labeled two secondary antibodies, specific for both the monoclonal and the polyclonal a-TNNI3, clearly showing that the unspecific interaction in case of the polyclonal antibody is higher than that of the monoclonal one $(\Delta \mathrm{F}$ refers to fluorescence counts).

seen in Figure 3 (a), the affinity binding of aTNNI3 antibody at the sensor surface does not change the fluorescence signal kinetics (step 2); however, the affinity binding of the respective secondary antibody leads to a strong change in the fluorescence signal $F(t)$. Firstly, rapid jump in fluorescence signal occurs due

to the excitation of Alexa Fluor 647 - labeled molecules in the bulk followed by gradual slower increase that is ascribed to the affinity binding of secondary antibody at the sensor surface. Upon the subsequent rinsing step, rapid decrease in the fluorescence signal is observed due to replacing the solution with fluorophore-labeled molecules from the flow cell and then a slow decay in the signal is attributed to the dissociation of the attached biomolecules and bleaching of the fluorophore labels at the sensor surface. The fluorescence sensor response $\Delta F$ that is associated with the affinity binding (defined as the difference between the baseline signal before the injection of a-TNNI3 and secondary antibodies and after the final rinsing) is linearly increasing with the concentration of the a-TNNI3. The unspecific interaction of the secondary antibody with the sensor surface (without captured a-TNNI3 antibody) was tested and a negligible signal of $\Delta F=132 \mathrm{cps}$ was measured for the anti-mouse secondary antibody (specific for the monoclonal antibody), however, a strong response of $\Delta F=2790$ cps was observed for the anti-goat secondary antibody (specific for the polyclonal antibody), as shown in Figure 3(b). As was expected, the SPFS readout using a sandwich assay was not sensitive enough for low concentrations, due to the close proximity of the epitopes aa 87-91 and aa 30-90 (Figure 1), therefore an inhibition-competitive assay format was developed for the detection of cTnI analyte using the monoclonal a-TNNI3 antibody. Although polyclonal antibodies, containing accurately identified antigenic determinants, can provide an alternative to monoclonal antibodies for the detection of diseases biomarkers, whenever multipoint binding of the antigen is offered, which can result in an increased sensitivity of the assay due to the increase in the avidity of antibodyantigen interaction [13], monoclonal antibodies are more advantageous than polyclonal ones. Their specificity is the most important advantage, as they only react with one epitope on the target molecule, while polyclonals contain hundreds or even thousands of specificities that can lead to cross 
reactions and consequently much less sensitivity. In addition to that, our SPFS results clearly indicate that the nonspecific binding in case of the polyclonal a-TNNI3 antibody is much higher (Figure $3 b$ ), therefore we chose to develop the competitive assay using the monoclonal a-TNNI3. Given that cTnI is released into the circulation of patients of AMI predominantly in its complex form, it is crucial to use antibodies which can recognize, not only free cTnI but also cTnI complexed with other cTn subunits [31], monoloclonals would be more advantageous in that regard. a mixed thiol SAM by amine coupling. Secondly, the analyzed sample with cTnI was spiked with mouse monoclonal a-TNNI3 antibody at a concentration of $2 \mathrm{nM}$ and reacted for $20 \mathrm{~min}$. Afterwards, the sample was flowed over the sensor surface for $20 \mathrm{~min}$ followed by the rinsing for $5 \mathrm{~min}$. Thirdly, a secondary anti-mouse antibody solution (6 $\mathrm{nM}$ ) was reacted for 20 min with the affinity bound a-TNNI3 antibody at the sensor surface. After rinsing for $5 \mathrm{~min}$, the fluorescence sensor response $\Delta \mathrm{F}$ was determined. The established calibration curve in Figure 4 (b) shows that the
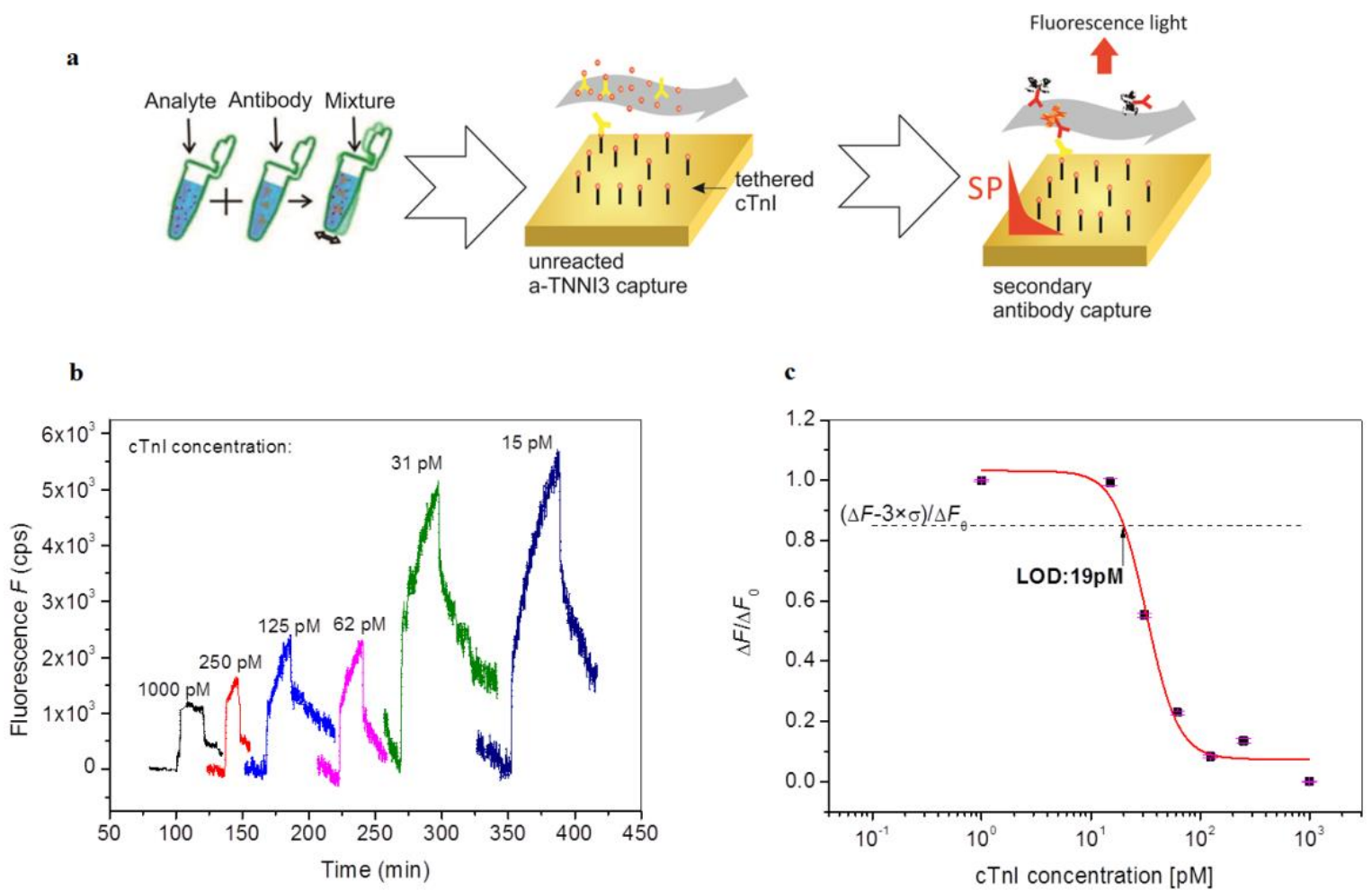

Figure 4. (a) A schematic of the inhibition-competitive assay steps, (b) fluorescence signal kinetics measured upon the binding of the secondary antibody conjugated with Alexa Fluor 647 and varied concentrations of cTnI, (c) normalized calibration curve of the SPFS biosensor with indicated limit of detection of $19 \mathrm{pM}$.

As schematically shown in Figure 4 (a), the developed cTnI assay with SPFS readout consisted of three steps. Firstly, a sensor chip was prepared with $\mathrm{cTnI}$ protein immobilized to sensor response $\Delta \mathrm{F}$ is inversely proportional to the target cTnI analyte concentration. In a sample with low amounts of cTnI analyte, the majority of binding sites of a-TNNI3 antibody 
spiked into the sample are free and can react with cTnI coupled to the sensor surface. Therefore, large fluorescence signal is generated after the reaction with the secondary antibody. For large amounts of cTnI target analyte molecules present in the sample, the binding sites of a-TNNI3 antibody are occupied, and do not bind to the surface, thus leading to a low fluorescence signal response. In order to calculate the limit of detection, the response of the fluorescence signals was plotted as a function of the cTnI analyte concentrations [Figure 4 (c)], and the curve was fitted using the sigmoidal function [3233]:

The achieved limit of detection (LoD) is 19 $\mathrm{pM}$, it was determined as the concentration for which the fitted calibration curve intersects the fluorescence signal measured for a blank sample $(\triangle \mathrm{F} 0=132 \mathrm{cps})$ lowered by three times the standard deviation of noise $(\sigma=10.6 \mathrm{cps})$. Although this achieved LoD is not the lowest among the previously investigated detection methods of cTni, it is to the authors' knowledge, the first time to detect this protein using a single monoclonal antibody specific for one epitope, which would be very beneficial to use in case of using real samples where the protein is known to be in its complex form. Detailed summaries of the other detection methods and LoDs are in references $[10,34]$.

It is worth of noting that the proposed SPFS readout is inherently more tolerant to fouling of the sensor surface that inevitably occurs on the gold sensor surface with thiol selfassembled monolayer carrying oligo(ethylene glycol) chains when realistic samples such as blood serum [35] are tested. For the analysis of biomarkers in more complex samples including saliva and blood plasma, more advanced biointerfaces based on anti-fouling polymer brushes [36-37] can be deployed on the surface of plasmonic biosensors as was previously reported by our laboratory. In combination with the advancement of SPFS instrumentation and biointerfaces (which were not the subject of the present research reported here), the reported results make an important step towards developing affinity biosensors that can serve for rapid diagnosis of cardiac diseases at an early stage with reliable and reproducible results.

In conclusion, we have developed sensitive troponin cTnI immunoassay with plasmonically amplified fluorescence readout, using a single monoclonal antibody, with an LoD of $19 \mathrm{pM}$ in 45 min detection time. It takes advantage of surface plasmon-enhanced fluorescence - SPFS - detection on the sensor surface that is optically probed by plasmonically enhanced optical field intensity at the fluorophore label absorption wavelength. We illustrate that the direct detection format based on regular surface plasmon resonance - SPR - biosensor principle does not provide sufficient sensitivity for the analysis of cTnI at clinically relevant concentrations. By using same instrument operated in SPFS modality, we significantly improved the sensitivity by implementing the inhibition-competitive immunoassay. This assay format is only allowed by careful selection of cTnI epitope and respective monoclonal antibody based on both experimental trials and theoretical modeling. These studies confirmed that immobilizing the ligand (recombinant version of target cTnI protein) directly to the surface via its N-terminal leaves the epitopes aa 30-90 and aa 87-91 available for affinity binding of respective immunoglobulin $\mathrm{G}$ antibodies.

\section{Methods}

Materials. Recombinant cTnI protein TNNI3 and anti-(TNNI3) goat polyclonal antibody were purchased from MyBioSource, San Diego, CA, USA (cat \# MBS2010502 and cat 
\# MBS 833132 respectively). The TNNI3 protein was reconstituted in phosphate buffer

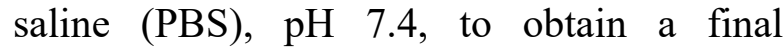
concentration of $100 \mu \mathrm{g} / \mathrm{ml}$. High performance chromatography (HPLC) was done to purify and remove any salts from the protein. Monoclonal mouse IgG against TNNI3 epitope aa 87-91. Goat anti-mouse IgG conjugated with Alexa Flour 647, and Donkey anti-goat IgG Alexa Flour 647, were purchased from antibodies-online, USA (Catalog Num.:A-21447).

Gel electrophoresis. 1D SDS PAGE was performed for the full-length recombinant TNNI3 to determine purity and molecular weight [13]. $5 \mu$ of pure sample was mixed with $5 \mu$ sample buffer [13] (156 mM TRIS, $5 \%$ SDS, $25 \%$ glycerol, $0.5 \%$ bromophenol blue, and 12.5\% 2-mercaptoethanol) [13]. The mixture $(10 \mu \mathrm{l})$ was loaded on a Bolt $4-12 \%$ Bis-Tris Plus Gels, $1.0 \mathrm{~mm} \times 15$ well (Life Tech-nologies Europe, Bleiswijk, the Netherlands). Gels were run at a constant voltage of $200 \mathrm{~V}$ for $50 \mathrm{~min}$ in MOPS buffer (0.025 M MOPS, 0.025 M TRIS, $3.465 \mathrm{mM}$ SDS, $1.025 \mathrm{mM}$ EDTA) using the XCell SureLock Mini Cell electrophoresis chamber (Invitro-gen, Karlsruhe, Germany). For all experiments Low range marker (MyBioSource, USA) was used [13]. Gels were stained using colloidal Coomassie Brillant Blue R-250 (MP, BIO, ICN, Santa Ana, CA, USA; cat \# 821616).

Western blot. After performing the full length protein to 1D SDS PAGE as explained above, samples were blotted onto a PVDF membrane [13] (Ji'an Qingfeng membrane Co., Ltd, Ji'an, China) by semi-dry blotting for $1 \mathrm{~h}$ with an electric current of $1.2 \mathrm{~mA} / \mathrm{cm} 2$, followed by cutting the membrane into strips and blocking with $2 \mathrm{ml}$ buffer (TBS 5\% nonfat dry milk powder, 1\% BSA) and incubation for $2 \mathrm{~h}$ [13]. The goat anti-TNNI3 polyclonal antibody (suspend-ed in 0.01 M PBS [13], pH 7.4, concentration: $1 \mathrm{mg} / \mathrm{ml}$ ) was used after
1:1800 dilution with blocking buffer [13]. Each strip was blocked and then incubated with $2 \mathrm{ml}$ polyclonal antibody solution for 24 $\mathrm{h}$ at $4^{\circ} \mathrm{C}$. This step was followed by three washing steps using $2 \mathrm{ml}$ washing buffer per strip (TBS, 0.05\% TWEEN 20 (v/v), 0.1\% BSA (w/v)) [13]. The secondary antibody used for western blot was anti-goat IgG + HRP from MyBioSource cat \# MBS 440120). The antigoat IgG + HRP secondary antibody solution was diluted 1:20,000 with blocking buffer, then added, $2 \mathrm{ml}$ per strip, and incubated for 1 $\mathrm{h}$ at room temperature [13]. This was followed by a last washing step and then visualization of the resulting band using $\mathrm{CN} / \mathrm{DAB}$ substrate kit (Thermo Fisher Scientific Pierce Biotechnology, MA, USA, cat \# 34000).

Sensor chip preparations. $50 \mathrm{~nm}$ gold layer was deposited on a high refractive index glass coated with $1.5 \mathrm{~nm}$ chromium, the gold layer was chemically modified with a mix of thiol with Carboxy group and thiol with $\mathrm{OH}$ group with ratio 1:9 (Sigma) by incubation overnight. The chips were then mounted on a sample holder while being attached to a flow cell of $\sim 4 \square \mathrm{L}$ volume. The thiol layer was activated by a mix of EDC/ NHS (1:1 ratio) in order to convert the $\mathrm{COOH}$ group to $\mathrm{NH} 2$ group, this was done in-situ using a pump at a flow rate $(40 \mu 1 / \mathrm{min}$.).

SPR and SPFS measurements. An optical system combining surface plasmon resonance (SPR) and surface plasmon enhanced fluorescence spectroscopy (SPFS) was used for direct investigation of the interaction between the protein and its antibodies. The Kretschmann configuration was used for the detection; a sensor chip with $50 \mathrm{~nm}$ gold layer on the top was optically matched to the base of high refractive index glass prism. A laser beam at wavelength of $633 \mathrm{~nm}$ was coupled to the prism and allowed to resonantly excite surface plasmons at the outer interface of thin gold film with a strength that was controlled by the angle of incidence. 
A flow cell with a volume of $4 \square \mathrm{L}$ was clamped against the gold sensor surface in order to flow liquid samples with a flow rate of $40 \mu \mathrm{L} / \mathrm{min}$. The reflected beam intensity was measured with a lock-in amplifier in order to track changes in the SPR signal. The fluorescence signal measured on the SPFS modality was excited via the enhanced electromagnetic field intensity generated by surface plasmons. The emitted fluorescence light from the sensor surface was collected through the flow cell by a lens with a numerical aperture about $\mathrm{NA}=0.2$ and detected by a photomultiplier (H6240-01, Hamamatsu, Japan) that was connected to a counter. The intensity of the excitation beam irradiating the sensing area on the sensor chip of about $1 \mathrm{~mm} 2$ was reduced to $30-60 \mu \mathrm{W}$ to decrease bleaching of Alexa Fluor 647. The fluorescence light emitted at a wavelength of about $670 \mathrm{~nm}$ was spectrally separated from the excitation light at $633 \mathrm{~nm}$ by using a set of laser notch filter and fluorescence band pass filter

\section{List of the SPFS set up devices:}

Device Supplier

Counter (53131A) Agilent (Santa Clara, USA)

Fluorescence band pass filter (670FS10-25) Andover Corporation Optical Filter (Salem, USA)

Laser notch filter (XNF-632.8-25.0M) CVI Melles Griot (Albuquerque, USA) Lockin amplifier EG\&G (Gaithersburg, USA)

Photomultiplier (H6240-01) Hamamatsu

Photonics (Hamamatsu, Japan)

Protein structure modeling. Protein structure modeling aims to determine spatial location of every atom in the protein molecule from the amino acid sequence by computational calculations. This was done using I- TASSER server [38-40]. The server uses I-TASSER based algorithms to automatically generate high-quality model predictions for 3D protein structure from their amino acid sequences. The I- TASSER detects structure templates from the Protein Data Bank using fold recognition technique, which is based on protein modeling using known protein structures. The full-length structure models are constructed by reassembling structural fragments from the protein folding templates using replica exchange Monte Carlo simulations [41].

\section{AUTHOR INFORMATION Corresponding Author \\ * Emails: \\ Amal.Kasry@bue.edu.eg, Jakub.Dostalek@ait.ac.at, Reham.Kased@bue.edu.eg}

\section{Author Contributions}

R.F.E. performed the SDS PAGE, Western blot analysis and the protein modelling, A.B., V.J., and A.K. designed and performed the SPR experiments. A.K., A.B., R.F.E. wrote the paper, J.D. and W.K. contributed to both the design of the experiments and writing the paper. All authors contributed to different aspects of the work, including writing and reviewing the paper.

\section{Notes}

The authors declare no competing financial interest.

\section{ACKNOWLEDGMENT}

The authors would like to thank Competence Centre for Electrochemical Surface Technology (CEST) and the British University in Egypt (BUE) for the financial support. JD acknowledges a support from European Union's Horizon 2020 research and innovation program under the Marie Sklodowska-Curie grant agreement no. 64268 . 


\section{REFERENCES}

1. WHO. Cardiovascular diseases. Available at: https://www.who.int/cardiovascular diseases/ about cvd/en/

2. WHO. Cardiovascular diseases. Available at: http://www.who.int/mediacentre/fact sheets/fs317/en/. (Accessed September 4, 2018).

3. Babuin, L.; Jaffe, S. A. Troponin: the biomarker of choice for the detection of cardiac injury, CMAJ, 2005, 173, 1191-1202. 4. Thygesen, K.; Alpert, J. S.; White, H. D. Universal Definition of Myocardial Infarction. Eur. Heart J. 2007, 28, 2525-2538. 5. Nusier, M.K.; Ababneh B.M. Diagnostic efficiency of creatinine kinase (CK), CKMB, troponin T and troponin I in patients with suspected acute myocardial infarction, J. Health Sci. 2006, 52, 180-185.

6. Forberg, J. L.; Henriksen, L. S.; Edenbrandt, L.; Ekelund, U. Direct Hospital Costs of Chest Pain Patients Attending the Emergency Department: A Retrospective Study. BMC Emerg. Med. 2006, 6, 1-7.

7. Wendy, L.; Cook, D.J.; Griffith, L.E.; Crowther, M.A.; Devereaux, P.J. Elevated cardiac troponin levels in critically ill patients: prevalence incidence and outcomes, Am. J. Crit. Care, 2006, 15, 280-288.

8. Heidenreich, P.A et al. The prognostic value of troponin in patients with non-ST elevation acute coronary syndromes: a metaanalysis, J. Am. Coll. Cardiol. 2001, 38, 478485.

9. Qureshi, A.; Gurbuz, Y; Niazi, J. H. Biosensors for cardiac biomarkers detection: A review. Sens. Actuators B, 2012, 171, 6276.

10. Han, H.; Li, S.; Peng, P.; Othman, A.M.; Leblanc, R. Recent Development of Cardiac Troponin I Detection. ACS Sens, 2016, 1, 106-114.
11. Katrukha, AG. Antibody selection strategies in cardiac troponin assays. in Cardiac Markers, 2nd edition (ed Wu, A.H. B.), Springer, 2003, 173-185.

12. Vylegzhanina, A. V.; Katrukha, I. A.; Kogan, A. E.; Bereznikova, A. V. Epitope Specificity of Anti-Cardiac Troponin I Monoclonal Antibody 8I-7. Clin. Chem. 2013, 59, 1814-1816.

13. El-kased, R. F. Immuno-analytical approach and its application for cardiac disease marker detection. J Immunoassay Immunochem, 2018, 39, 538-550.

14. Sanjay, S. T. et al. Biomarker detection for disease diagnosis using cost-effective microfluidic platforms. Analyst, 2015, 140, 7062-7081.

15. Apple, F. S.; Smith, S. W.; Pearce, L. A.; Murakami, M. M. Assessment of the Multiple- Biomarker Approach for Diagnosis of Myocardial Infarction in Patients Presenting with Symptoms Suggestive of Acute Coronary Syndrome. Clin. Chem. 2019, 55, 93-100.

16. Knoll, W. Interfaces and thin films as seen by bound electromagnetic waves. Ann. Rev. Phys. Chem. 1998, 49, 569-638.

17. Liebermann, T.; Knoll, W. Surfaceplasmon field-enhanced fluorescence spectroscopy. Colloids Surf. 2000, 171, 115130.

18. Knoll, W. et al. New concepts with surface plasmons and nano-biointerfaces, JNOPM, 2008, 17, 121-129.

19. Dostálek, J.; Kasry, A.; Knoll, W. Long range surface plasmons for observation of biomolecular binding events at metallic surfaces. Plasmonics, 2007, 2, 97-106.

20. Yang, C.T.; Wu, L.; Liu, X.; Tran, N. T.; Bai, P. et al. Explointing Surface-PlasmonEnhanced light scattering for the design of ultrasensitive biosensing modality. Anal. Chem. 2016, 88, 11924-11930.

21. Martin, B.; Koji, T.; Mana, T.; Qingwen, Z.; Jakub. D. Plasmon-Enhanced fluorescence biosensors: a Review. Plasmonics, 2014, 9, 781. 
22. Tadepalli, $\mathrm{S}$. et al. Peptide Functionalized Gold Nanorods for the Sensitive Detection of a Cardiac Biomarker Using Plasmonic Paper Devices. Sci Rep. 2015, 5, 16206.

23. Liu. X. et al. Peptide Functionalized Gold Nanoparticles with Optimized Particle Size and Concentration for Colorimetric Assay Development: Detection of Cardiac Troponin I. ACS Sensors, 2016, 1, 1416-1422.

24. Yang, C. T. et al. Exploiting SurfacePlasmon-Enhanced Light Scattering for the Design of Ultrasensitive Biosensing Modality. Anal. Chem. 2016, 88, 11924-11930.

25. Aslan, K.; Grell, T. A. J. Rapid and Sensitive Detection of Troponin I in Human Whole Blood Samples by Using Silver Nanoparticle Films and Microwave Heating. Clin Chem. 2011, 57, 746-752.

26. Qureshi, A.; Gurbuz, Y.; Niazi, J.H. Biosensors for cardiac biomarkers detection: A review, Sens. Actuators B, 2012, 171-172, 62-76.

27. Guy, M.J.; Chen, Y.C.; Clinton, L.; Zhang, H.; Zhang, J. et al. The Impact of Antibody Selection on the Detection of Cardiac Troponin I. Clin Chim Acta. 2013, 420, 82-88.

28. Cai, Y.; Kang, K.; Li, Q.; Wang, Y.; $\mathrm{He}, \mathrm{X}$. Rapid and Sensitive Detection of Cardiac Troponin I for Point-of-Care Tests Based on Red Fluorescent Microspheres. Molecules, 2018, 23, 1102.

29. Fischer, M.J. E. Amine Coupling Through EDC/NHS: A Practical Approach. in Surface Plasmon Resonance; Methods and Protocols, Methods Mol Biol. 2010, 627, 5573.

30. Peronnet, E.; Becquart, L.; Martinez, J.; Charrier, J.P; Jolivet-Reynaud, C. Isoelectric point determination of cardiac troponin I forms present in plasma from patients with myocardial infarction. Clin Chim Acta, 2007, 377, 243-247.

31. Katrukha, A.G.; Bereznikova, A.V.; Esakova, T.V.; Pettersson, K.; Lövgren, et al.
Troponin I is released in bloodstream of patients with acute myocardial infarction not in free form but as complex, Clin. Chem. 1997, 43, 1379-1385.

32. Wang, Y.; Dostalek, J.; Knoll, W. Long range surface plasmon-enhanced fluorescence spectroscopy for the detection of aflatoxin M1 in milk. Biosens. Bioelectron, 2009, 24, 2264-2267.

33. Dostalek, J.; Pribyl, J.; Skladal, P.; Homola, J. Multichannel SPR biosensor for detection of endocrine disrupting compounds, Anal. Bioanal. Chem, 2007, 389, 1841-1847.

34. Upasham, S.; Tanak, A.; Prasad, S. Cardiac troponin biosensors: where are we now? Adv. Health Care Tech. 2018, 4, 1-13.

35. Hageneder, S.; Bauch, M.; Dostalek. J. Plasmonically amplified bioassay - Total internal reflection fluorescence vs. epifluorescence geometry, Talanta, 2016, 156157, 225-231.

36. Riedel, T.; Hageneder, S.; Surman, F.; Pop-Georgievski, O.; Noehammer, C. et al. Plasmonic Hepatitis B Biosensor for the Analysis of Clinical Saliva, Anal. Chem. 2017, 89, 2972-2977.

37. Kotlarek, D.; Vorobii, M.; Ogieglo, W.; Knoll, W.; Rodriguez-Emmenegger, C. et al. Compact Grating-Coupled Biosensor for the Analysis of Thrombin, ACS Sens. 2019, 4, 2109-2116.

38. Zhang, Y. I-TASSER server for protein 3D structure pre-diction. $B M C$ Bioinformatics, 2008, 9, 40.

39. Roy, A.; Kucukural, A.; Zhang, Y. ITASSER: a unified platform for automated protein structure and function prediction. Nat Protocols, 2010, 5, 725-738.

40. Yang, J. et al. The I-TASSER Suite: Protein structure and function prediction. Nat Methods, 2015, 12, 7-8.

41. Thachuk, C.; Shmygelska, A.; Hoos, H.H. Replica exchange Monte Carlo algorithm for protein folding in the HP model, $B M C$ Bioinformatics, 2007, 8, 342. 